\title{
Research on the development space of urban leisure sports in China
}

\author{
Fan Zhang \\ Department of Police Skills and Tactics, Nanjing Forest Police College, Nanjing 210023, China \\ Sports Science postdoctoral programme, Nanjing Normal University, Nanjing 210023, China \\ zhangfan@nfpc.edu.cn
}

Keywords: sports management, leisure sports, national fitness program, city, development space.

\begin{abstract}
The comparative analysis and logical analysis are utilized to study the development space of urban leisure sports in process of urbanization in China. Main conclusions: In the five-year plan period from 2016 to 2020, China's society has entered a deep development stage. With the deepening urbanization and continuous development of national fitness, the development space of urban leisure sports should shift the work focus to the upgrading of leisure sports consumption, and demands of middle-class families and adolescents. The differentiation and ecology of leisure sports competition attributes will also become the new trend of urban leisure sports development in the future.
\end{abstract}

\section{Introduction}

The National Fitness Program (2016-2020) has not only made comprehensive and definite progress in terms of overall requirements, main tasks, safeguards, organization and implementation compared to previous two plans, but also is an important measure of central government to administer sports departments, govern sports undertakings, promote public sports services, national fitness and industrial growth. At the same time, it is also regarded as an important grasp to meet the growing health and spiritual and cultural needs of citizens in urbanization process.After 30+ years of reform and openness, China's urban development and urbanization rate have been greatly improved. The data from National Bureau of Statistics indicates that China's urbanization rate has increased from $17.92 \%$ in 1978 to $54.77 \%$ in 2014. However, the sharp increase of urban population has also brought a series of "urban diseases" e.g. congestion, environmental damage, sharp decrease in per capita resources; of which the effective supply of public sports resources that lagged behind the economic development becomes a particularly prominent problem in urbanization process. It has become a topic worthy of participation of urban managers and urban residents to nurture urban residents and create better life through urban development. Based on academic perspective of urban governance and urban planning, this paper studies the change of urban residents' leisure demands in the future, especially the development space of leisure sports. In the deep development stage of the National Fitness Program, combined with the construction of urban public sports service, this paper studies the service of urban sports planning and urban construction in a more forward-looking way in order to enter the sustainable development process of "urban construction pleases residents, residents serve urban construction".

\section{Background of National Fitness Program to develop in depth and breadth}

With the deepening of reform and openness as well as overall economic growth, the development of cities and modernization process have brought great changes to people's lives. The demands for diversified lifestyles and life contents constantly impact city's construction planning, governance concepts and service levels. In order to match the progress and development of modernization cause, a new mass sports creed and concept, i.e. the National Fitness Program for the purpose of strengthening people's physique, have come into being.

The Outline of National Fitness Program (hereinafter referred to as the program outline) reflects 
the social (sports) management concept of government under the era background. By examining the program outline, it can clearly understand the imprint and characteristics of sports management at that time, which reflects the characteristics of sports management since the founding of New China in the past decades to a certain extent - the underestimate on the mass sports work under the national system and Olympic glory [1]. The program outline decomposes the work objectives: in the first five years, efforts should be made to improve the environment and conditions for mass sports and fitness activities; in the next ten years, a national fitness system with Chinese characteristics should be basically established, and the sports reform should be deepened. Combined with historical documents, it can find that China's sports reform did make progress at the end of 20th century, and great changes have taken place in the national sports administration departments: the State Sports Commission has been transformed into the State Sports General Administration. The introduction of three big ball market mechanism has brought all-round development to competitive sports. However, the dividend of sports reform has not brought great benefits to national fitness, on the contrary, the objectives of program outline were still very general, there was no comprehensive and clear development plan in 15 years of development. Most of the development objects and key work, including countermeasures and organizational measures, etc., belonged to literal propaganda work and lack specific explanation and guidance; standardized, scientific, standardized and assessed work requirements didn't form. Although the promulgation of program outline reflects the awakening of times and is a progress in concept of sports reform, it also illustrates the backwardness of government's sports management concept, lack of understanding and deficient sports scientific research under the era background.

After the development of three five-year plans (1996-2010: Ninth Five-Year Plan, Tenth FiveYear Plan and Eleventh Five-Year Plan), the national economy, per capita income, concept of national governance, sociological research, cultural undertakings, etc, have made tremendous changes. Especially the sports industry, after hosting a series of major sports activities e.g. Beijing Olympic Games, the city's enthusiasm for sports has become strong, many cities have generated brand sports events [2, 3]. Urban residents' enthusiasm for participating in physical exercise has been greatly enhanced; sports scientific research has also made great strides forward, which has also made great contributions to the development of national, regional and local sports. In this context, the State Council issued the National Fitness Plan (2011-2015) in February 2011 to further plan and guide the national fitness cause in the post-Olympic period. The National Fitness Program (20112015) has made a qualitative leap and improvement over the previous one. Its guiding ideology is based on building a affluent society in an all-round way and socialist spiritual civilization, serving the coordinated development of competitive sports and national fitness, and encouraging people to gain all-round development through participating in sports. In terms of goals and tasks, it is comprehensively clear and targeted; many tasks even have a clear and measurable description. For example, the number of people who often participate in physical exercise has further increased: not less than three times a week, not less than 30 minutes each time; proportion of people with moderate exercise intensity reached over 32\%, $3.8 \%$ higher than that in 2007. There were more than 1.2 million stadiums and more than 1.5 square meters per capita sports area in China. The work measures were appropriate, reasonable, orderly, with prominent features. The safeguard measures pointed out a new method for funds raising and provided a new direction for development of capital service sports. In the organization and implementation link, the people's governments at or above the county level were required to participate in it, and should put the national fitness program on an important agenda and make a comprehensive evaluation. This is an important progress for the program outline, which reflects government's efforts in achieving progress in social governance and orderly implementation of national fitness work.

On June 23, 2016, the National Fitness Program (2016-2020) was officially released and became a hot topic in society, which would cause significant impacts on the development of sports in China during 13th Five-Year Plan. New plan is more profound in guiding ideology, which not only serves people's physical and health level, but also bases on state's modern governance capacity and working principles, and thus serves the construction of affluent society in an all-round way and 
through overall planning, establishment of public service system and ecological industrial chain. The development goal is more clear, the number of participants in physical exercise and the promotion of sports consumption are predicted and required; and a clear pattern of development of the national fitness program has formed. Compared to the primary mission of 2011-2015, the plan focus of 2016-2020 is prominent, which fits the 13th Five-Year Plan of National Economy, i.e. to enrich supply, promote reform, construct facilities, form pattern, expand exchanges, foster culture, equalize and stress the essentials. In order to achieve the goal, the plan has made great efforts in safeguard measures; it starts from the working mechanism, fund guarantee, evaluation system, incentive mechanism, scientific and technological innovation, talent construction, legal policy and other links, and then truly realizes the national fitness pattern. In the aspect of organization and implementation, the national fitness work is included in the annual livelihood of local governments at all levels to promote and assess, and the evaluation system is introduced for comprehensive evaluation, third-party evaluation, special evaluation and satisfaction survey. Supplemented by multi-monitoring mechanism, it aims to practically promote the state strategy of national fitness.

These three national fitness programs reflect the great progress of government's social governance to a certain extent, especially the efforts of new central government in promoting people's livelihood and implementation. The great progress of the National Fitness Program (20162020) not only lies in the details of tasks, but also the implementation and assessment, which ultimately allows plan to serve people's life, health, integrated development and happy life. At the same time, by enhancing physical fitness and happiness, it gradually enters the sustainable cycle of serving social progress. National Fitness Program is an important component and a powerful supplement to the national economic and social development plan. Through the comprehensive comparative study on three national fitness programs and social development, it is concluded that the current National Fitness Program (2016-2020) has entered a stage of in-depth development, i.e. the stage that serves the overall development of people and serves the social progress.

\section{Basic theory of urbanization and leisure sports}

\subsection{Urbanization and leisure sports}

Cities emerge with the evolution of human settlements, and show the characteristics of modernization with the process of industrialization: integrating multiple elements or some elements such as regional politics, economy, culture, etc. Cities and villages are two manifestations of human settlements, which are integrated and antagonistic. Professor Luo Chun (2013) believes that urban morphology is also evolving: according to the evolution stage of urbanization, urban morphology can be divided into towns, cities and metropolis. These three belong to the non-agricultural population-gathering places but show different settlement patterns. Cities and towns have small population concentration and regional scale, with certain country flavor; there have space expansion, population growth, opposite and prominent contradiction between urban and rural areas in cities; metropolis are characterized with overall spatial expansion, highly aggregated population and highlighted city-regions. These three patterns also reflect the initial, middle and late stages of urbanization.

A dominant sign of urbanization evolution is the change of urban population proportion (urbanization rate). When the outline of national fitness program was released in 1996, China's urbanization rate was 30.48\%, 36.22\% in 2000 (the first stage of mission was completed (2000), and 49.95\% in 2011-2015 (National Fitness Program was released (2011-2015). 50\% of urbanization rate was internationally-recognized watershed. Over 50\% meant that the urban and rural pattern in China would be reversed, the traditional Chinese rural society would gradually drift away. However, just in second years (2011), China's urbanization rate has exceeded 50\% and reached $51.27 \%$. The latest demographic statistics (2014) showed that China's urbanization rate has reached $54.77 \%$. China has entered the mid-stage of urbanization; urban space and scale has increased sharply; urban population has grown; the second industry and the tertiary industry expanded and showed the pressure of industrial transformation. 


\subsection{Urbanization and leisure sports}

Sports humanities and sociology circles has extensively discussed the "leisure sports" from both "logical starting point theory" or "realistic concern theory". By studying the mutual stipulation between sports and leisure, Li Rui (2016) believes that leisure opens new sport, and sports rectifies the name of leisure; he also interprets leisure sports from the perspective of realistic concern, which has strong philosophical thinking characteristics. This study tends to agree with Professor $\mathrm{Li}$ Xiangru's (2011) explanation that leisure sports are a kind of self-conscious and sufficient social cultural activity in which people can meet their physical and mental needs through direct or indirect experience in spare time and by means of sports [4].

In the process of urbanization, urban residents promote the process of urban modernization through high-load and efficient work. With the development of urbanization, they begin to enjoy the development results of urbanization. Use of leisure disposable time and participation in sports to meet physical and mental needs have become the basic living conditions and needs for urban people. Higher level of urbanization and more number of urban population cause reduction of per capita sports resources. Especially in the current situation of insufficient effective supply of urban sports resources, urban people's demand to participate in leisure sports is suppressed, the enthusiasm and effect of participation are affected. National Fitness Program (2016-2020) has the task and implementation strategy to solve the problem of participating in exercise and enriching sports supply. In this process, through appropriate resources allocation, government behavior and social supplement, it has far-reaching significance to effectively improve the leisure sports needs of a large number of urban residents.

\subsection{Development space of urban leisure sports}

Studying the development space of urban leisure sports is aimed to improve the leisure sports life space of urban residents. Chang Naijun (2011) believes that urban leisure sports living space is "a place for leisure sports activities in the city". He divides urban residents' leisure space into physical space and social cultural environment; of which the material space environment includes the place space and place environment; the social culture environment includes the activity place management, the sports activities atmosphere and local sports culture. As urban residents' participation in leisure sports is closely related to living space, it can pay close attention to their leisure sports living space and study the changes of leisure sports living space in the process of urbanization so as to better serve the development of urban leisure sports in the next few years or years through rational planning and resource allocation.

\section{Development space of China's urban leisure sports in deep development process of national fitness}

\subsection{The effective supply of leisure sports activities will promote urban residents' consumption for leisure sports.}

The National Fitness Program (2016-2020) clearly states that it should carry out fitness activities around the masses according to time, region and needs, guide the development of sports items in different levels and classifications, enrich and improve the system of national fitness activities. It should not only vigorously carry out popular sports, but also promote the development of fashionable leisure sports with consumption-leading characteristics and traditional folk sports. For example, Yangzhou has opened more than three fencing clubs in past two years. Luoyang has opened more than 10 archery clubs [5]. The clubs that golf club provides indoor golf simulator operation reaches nearly 300 . With the abundant supply of these leisure sports items with obvious consumption characteristics, urban people's participation choice in leisure sports has greatly increased, and sports consumption will also reach a higher level and promote the development of urban leisure sports base industry. With the popularization of leisure projects and venues, the matching leisure sports service industry will bring greater industrial value and development potential, e.g. leisure sports education and training, mass community-type leisure sports events. 


\subsection{Huge differentiation has appeared in urban leisure sports: the middle class family may be the main force of leisure sports consumption.}

With the continuous upgrading of urban residents' consumption for leisure sports, the development of leisure sports will automatically screen the participants in urban leisure sports. With the realization of building comprehensively affluent society and the further development of national economy, middle-class families will become the main force of consumption and urban consumption-oriented leisure sports. Golf, shooting, archery, fencing, racing, equestrian, aviation, water sports, ice and snow sports, etc. will be favored by middle class families [6]. Leisure sports within city, or suburbs, or even in city circle, will become a new choice for middle-class families. In addition, the sustainability and consistency of participating in leisure sports also require the economic conditions and time of middle-class families.

\subsection{The characteristics of urban leisure sports groups are more obvious: youth leisure sports consumption will become the core.}

The features of leisure sports participation groups will become more characteristic. The elderly and young people will become the main participation force of urban leisure sports. On the contrary, the middle-aged people will be involved for meeting the need of children and parents, and even more become the purchasers of leisure sports consumption. Combined with China's long-standing household consumption habits, we think that the proportion of the elderly becoming the main consumer of leisure sports in recent period is not high as they may prefer free leisure sports. In order to meet children's exercise needs or ever their education, some leisure sports with both educational attributes have better development potential, e.g. golf, equestrian and fencing.

\subsection{The competitive attributes of urban leisure sports are constantly differentiated - the distinction between competition and de-competition is obvious.}

With the development of leisure sports, the competitive attributes of urban leisure sports will be severely differentiated, and the strengthened sports items will become competitive items, e.g. basketball, football and other common sports; the development will effectively expand the reserve strength of professional sports, spectators and other categories so as to promote the development of professional sports in China. Those weakened sports will remove the de-competitive attributes and truly become nutritional leisure sports, e.g. yoga, Tai Ji and fishing.

\subsection{New development and new requirement of urban leisure sports -- ecology and sports proceed together.}

With the common development of economy and leisure sports, urban residents have higher requirements for leisure sports. With the diversification and popularization of supply, along with the upgrading and stimulation of industry, there will have a higher pursuit for leisure sports; the sports must achieve physical and mental development; the exercise environment must be beautiful and comfortable. The ultimate manifestation will be the pursuit of ecology. The combination of good ecological environment and physical exercise, and the integration of physical exercise with ecological environment and ecological civilization into physical and mental development of exercisers will become the new development and new objectives of urban leisure sports.

\section{Conclusion}

National Fitness Program has entered a deep development stage; government's social governance and sports management have also entered a new normal of "respecting people, loving people and serving people". Urbanization is a major trend to be faced and solved by China's economic and social development in a long time. National Fitness Program is a national strategic plan; it should be implemented through full investigation, reasonable planning and high-quality government guidance. New trend and space of urban leisure sports development should be studied to truly serve people's physical exercise and happy life. 


\section{Acknowledgements}

This work was supported in part by the Project of China Postdoctoral Science Foundation under Grant 2017M611849, in part by the Project of the Fundamental Research Funds for the Central Universities under Grant LGZD201805, in part by the 13th Five-Year plan project of Jiangsu Education Science under Grant C-c/2018/01/11, in part by Jiangsu Qing Lan Project under Grant 2017, and in part by Pre-research project of Nanjing Forest police College under Grant LGY201603 , and in part by Nanjing Forest police College Teaching Reform Project under Grant ZD18104.

\section{References}

[1] Zhao Lei, Wu Wenzhi. (2013) Heritage of Local Culture and Planning Urban Green Space System. Urban Development Studies, 9, 26-31.

[2] SONG Tienan. (2015) Form of Sports Leisure Space in City in China from the Analysis Theory of Space in City. Journal of Shenyang Sport University, 12, 6-11.

[3] JIN Yin-ri, YAO Song-ping, JIANG Zong-yue. (2012) the Empirical Research on Structure and Formation of Urban Leisure Sports Business District. Journal of shanghai physical eduction institute, 12, 37-40.

[4] You Songhui, Qin Haiquan, He Chenjue, et al. (2011) Interactive Relationship between Sports Leisure Industry and City Development. Journal of shanghai physical eduction institute, 4, 58-62.

[5] HU Ying, LI Cui-xia. (2015) Investigation and Analysis of Leisure Sports in a Park from Diachronic and Ecological Points of View. China Sport Science, 8, 16-28.

[6] Wu Zhijian, Wang Zhuying, and Song Yan Li Qing. (2018) the Meta Analysis on the Built Environment's Influence on the Physical Activity of the Elderly. Journal of Shanghai University of Sport, 1, 64-71. 